\section{Cureus}

Received 09/04/2018

Review began 09/05/2018

Review ended 09/07/2018

Published 09/13/2018

\section{(c) Copyright 2018}

Ali et al. This is an open access article distributed under the terms of the Creative Commons Attribution License CC-BY 3.0., which permits unrestricted use, distribution, and reproduction in any medium, provided the original author and source are credited.

\title{
Managing Chronic Pain in the Elderly: An Overview of the Recent Therapeutic Advancements
}

Asad Ali ${ }^{1}$, Abdul Wahab Arif ${ }^{2}$, Chandur Bhan ${ }^{3}$, Deepak Kumar ${ }^{4}$, Muhammad Bilal Malik ${ }^{5}$, Zohaib Sayyed ${ }^{6}$, Khawaja Hassan Akhtar $^{7}$, Malik Qistas Ahmad ${ }^{8}$

1. Internal Medicine, CMH Lahore Medical College and Institute of Dentistry, Lahore, PAK 2. Medicine, Shaikh Zayed Hospital, Lahore, PAK 3. Internal Medicine, Chandka Medical College Hospital, Larkana, PAK 4. Internal Medicine, Jinnah Postgraduate Medical Centre, Karachi, PAK 5. Internal Medicine, Shifa College of Medicine, Lahore, PAK 6. Pediatrics, Shaikh Khalifa Bin Zayed Al-Nahyan Medical and Dental College, Lahore, PAK 7. Internal Medicine, King Edward Medical University/Mayo Hospital, Lahore, PAK 8. Attock Medical Center, Attock City, Punjab, PAK

$\square$ Corresponding author: Malik Qistas Ahmad, kistasmalic@yahoo.com Disclosures can be found in Additional Information at the end of the article

\section{Abstract}

A majority of the elderly suffer from chronic pain that significantly alters their daily activities and imposes an enormous burden on health care. Multiple comorbidities and the risk of polypharmacy in the elderly make it a challenge to determine the appropriate drug, dosage, and maintenance of therapy. Opioids are the most commonly used agents for this purpose in the elderly. The aim of this article is to discuss both the current well-established therapies used for managing chronic pain in the elderly and also the emerging newer therapies.

Categories: Internal Medicine, Neurology, Pain Management

Keywords: chronic pain, opioids, non-steroidal anti-inflammatory drugs (nsaid), quality improvement, geriatrics, neuropathic pain, topical agents, chronic illnesses, targeted therapy, pain management

\section{Introduction And Background}

The demographic transition in the recent decades has resulted in a significant increase in the aging population around the world. This, in turn, has resulted in an increased prevalence of various problems faced by the elderly. Chronic pain is one such common ailment reported in the elderly that also poses a significant economic burden on health care. Chronic pain is defined as the pain that lasts for three to six months or more than expected [1]. Similar to the figures in the general population, the prevalence of chronic pain in the elderly also varies widely from $15.2 \%$ in Malaysia [2] to $69.8 \%$ in Germany [3]. This is even higher (up to 83\%) among the elderly in nursing homes [4]. Pain in the elderly may be underreported due to the misconception that it is a normal process of aging and also due to cognitive disturbances.

\section{Pain mechanism}

Various mechanisms are proposed for increased pain and pain sensitivity in the elderly. The first mechanism is due to physiological changes in the elderly like a decrease in neurotransmitters such as gamma-aminobutyric acid (GABA), serotonin, noradrenaline, and acetylcholine, decrease in the number of peripheral nociceptive neurons, increased pain thresholds, and reduced endogenous analgesic responses resulting in a paradoxical increase in the pain. Another reason is homeostenosis in aging, which is the loss of homeostatic reserve of 
various organ systems manifested as liver and renal function decline, decreased muscle mass and increased frailty leading to falls, decreased appetite, sleep disturbances, depression, delirium, agitation, and overall debility [5].

\section{Causes of chronic pain in the elderly}

Musculoskeletal disorders such as degenerative spine and arthritic conditions are the most common cause of chronic pain in the elderly. Other common causes of significance include neuropathic pain, ischemic pain, and pain due to cancer as well as its treatment [6]. Among elderly women, there is a high prevalence of vertebral compression fractures causing pain and discomfort.

\section{Challenges and barriers}

Chronic pain in the elderly is associated with an increased incidence of adverse outcomes, including functional impairment, falls, depression, and sleep disturbances. Pain management in older persons differs significantly from that in younger persons. Concomitant chronic illnesses make pain evaluation and treatment more difficult in the elderly. Also, older people respond differently to various therapies, usually with lesser efficacy and more severe adverse reactions, including additional risks of polypharmacy and addictions. In addition to this, the majority of elderly living in nursing homes have some degree of cognitive impairment, which has an impact on their ability to report pain resulting in inadequate pain assessment and management [7]. There is a lack of evidence-based guidelines for the treatment of chronic pain in the elderly as studies tend to focus more on younger adults. However, in the recent past, there has been an increase in the number of studies focused primarily on pain control in the elderly.

\section{Pain assessment}

Before outlining a treatment plan, a comprehensive assessment of the patient is essential for making a specific diagnosis and to ensure targeted therapy whenever possible. The functional status of the patient and the patient's ability to perform daily activities must be evaluated to determine the degree of independence, level of need for caregivers, as well as overall quality of life [6]. It is important to note that the majority of the elderly have multiple chronic illnesses that may affect an accurate assessment of pain as well as the efficacy of treatment [8]. For assessing pain intensity, the available data support methods like visual analogy scale (VAS), verbal descriptor scale, and numerical rating scale. The VAS is a reliable method used usually in clinical and research settings as it is simple, has minimal intrusiveness, and is ease to administer. Still, it should be used with caution in elderly patients as many of them report difficulty in completing the VAS, or give incomplete responses making it more difficult to give them scores [9]. For assessing the sensory, affective, evaluative, and miscellaneous components of pain, the McGill Pain Questionnaire used usually has proven evidence for validity, reliability, and discriminative abilities but not age-related components [10]. Of particular importance in the elderly are various tools used for pain assessment in cognitively impaired persons. Scales using pictures like the Wong-Baker FACES scale can be used for the elderly with limited cognitive ability. In elderly persons with advanced dementia, behavioral observation is needed to identify the presence of pain, for which tools like Pain Assessment in Advanced Dementia (PAINAD) and Checklist on Nonverbal Pain Indicators (CNPI) can be used [11].

\section{Therapeutics}

Various treatment options are available for chronic pain management in the elderly including either pharmacological or non-pharmacological measures or both combined. A comprehensive approach to dealing with common sequelae such as depression, isolation, and physical disability is considered effective. Non-pharmacological measures are considered particularly 
important in elderly patients as they have a lower frequency of adverse reactions compared with pharmacologic approaches and their benefit is usually enhanced when combined with drug strategies. Effective non-pharmacological approaches include physical therapy, cognitive behavioral therapy, and most importantly, patient and caregiver education interventions [12, 13], while pharmacological treatment modalities include non-opioid and opioid medications, pain modulating drugs, topical agents, and other newer therapies.

\section{Review}

\section{Treatment guidelines}

The most appropriate treatment guidelines for chronic pain in the elderly are by the American Geriatrics Society (AGS), which were first formulated in 1998 and recently updated with newer pharmacological approaches in 2009 [1]. According to these guidelines, treatment is initiated by a drug with least toxicity preferably by the oral route, scheduled dosing with short-acting drugs in case of episodic pain, and consideration of rational polypharmacy with drugs of complementary mechanisms of action whenever needed.

Due to significant overlap between chronic geriatric pain and pain due to cancer, the World Health Organization (WHO) recommendations for pain management listed below [14] are also followed by a few clinicians.

(1) giving drugs "by the clock"

(2) by mouth and

(3) following "'analgesic ladder" which includes:

a. Mild pain - the first choice is acetaminophen.

b. Mild to moderate pain or pain uncontrolled with acetaminophen - NSAIDs.

c. Pain refractory to NSAIDs - weaker opioid agonist.

d. For pain refractory to the previous plan, or severe pain - pure opioid agonist.

e. Adjuvant medication may be used for synergism with the previous medications.

\section{Current Therapeutics}

Acetaminophen: American Geriatrics Society (AGS) recommends acetaminophen as the firstline agent for mild to moderate chronic pain in the elderly [1] due to its favorable safety profile. The maximum daily dosage of acetaminophen allowed is $4 \mathrm{~g}$ with dose adjustments up to 50$70 \%$ required in patients with hepatic dysfunction. Hepatotoxicity is another major concern due to unintentional overdosage of acetaminophen, to avoid which proper patient counseling is required. One meta-analysis of seven randomized controlled trials showed superior efficacy of acetaminophen compared to placebo in reducing moderate pain but inferior to NSAIDs for both pain reduction and physical functioning [15]. Acetaminophen is an effective agent for relieving symptoms of osteoarthritis and low back pain [16]. However, it is less effective for chronic inflammatory pain (such as the pain associated with rheumatoid arthritis) than NSAIDs [17]. Also, NSAIDs have a better effect as short-term (e.g., six weeks) treatment for osteoarthritis pain and low back pain.

Non-steroidal anti-inflammatory drugs (NSAIDs): Oral non-steroidal anti-inflammatory drugs (NSAIDs) like naproxen, ibuprofen, diclofenac, and celecoxib can be used for chronic pain in the elderly when acetaminophen fails to control the pain effectively. NSAIDs are particularly helpful in treating an inflammatory type of pain where acetaminophen has limited efficacy. The American Geriatric Society recommends great caution while using NSAID therapy in the elderly due to its established gastrointestinal, renal, and cardiovascular side effects and also recommends giving NSAIDs preferably for only short periods of time during episodic flares. A study on drug reactions leading to hospitalizations in the elderly showed NSAID related side effects as the cause for hospitalization in $23.5 \%$ of the elderly [18]. Gastrointestinal toxicity 
(gastric complications causing iron-deficiency anemia) risk due to NSAIDs is dose-dependent, increases with age, and also increases with concomitant use of cardioprotective doses of aspirin, which is common in elderly. Nonacetylated NSAIDs like salsalate were found to have limited but not proven evidence of lower gastrointestinal toxicity compared to aspirin [19]. Gastrointestinal (GI) side effects were also found to be reduced but not completely eliminated with selective cyclooxygenase-2 (COX-2) inhibitor NSAIDs like celecoxib. However, the risk of other toxicities is still high with COX-2 inhibitors and warrants caution for elderly use [20]. Some COX-2 selective inhibitors were withdrawn from the market because of a higher incidence of cardiovascular events, including rofecoxib and valdecoxib [21]. Protection against GI toxicity is achieved to a certain extent with concomitant administration of proton pump inhibitor, H2 blocker, or misoprostol and, if required, Helicobacter pylori eradication therapy. A metaanalysis showed a superior cardiovascular safety profile of naproxen compared with other selective or nonselective COX inhibitor NSAIDs [22]. The overall decision regarding the administration of NSAIDs for chronic pain in the elderly must be individualized based on comorbidities and other risk factors.

Topical NSAIDs: Topical NSAIDs therapy comprises an important alternative to oral NSAIDs in the elderly because of its rare systemic side effects and very few cutaneous effects like rash or pruritis at the site of application. Topical NSAIDs are often used for knee or hand osteoarthritis (OA) related pain. Evidence from a recent randomized controlled trial showed the comparable efficacy of topical diclofenac sodium with that of oral NSAIDs in the treatment of knee osteoarthritis with fewer adverse effects [23]. This is because of increased delivery of drug to adjacent synovium without much systemic absorption. In the United States, there are two approved topical NSAID formulations for OA: diclofenac sodium topical solution 1.5\% in $45.5 \%$ dimethyl sulfoxide solution and diclofenac sodium $1 \%$ gel. Another category of drugs are rubefacients, which include salicylate-containing drugs like trolamine salicylate or methyl salicylate that act by counter-irritation, but they have a lesser effect on chronic pain control compared to topical NSAIDs and increased risk of salicylate toxicity [24].

Capsaicin: Capsaicin is an active alkaloid derivative from the capsicum family that acts by reduction of substance $P$ and cutaneous nociceptive fibers. It can be used for both neuropathic pain and musculoskeletal pain but mostly as an adjuvant analgesic. The major disadvantage of this therapy is a burning sensation, which is particularly high in patients with existing scarred skin conditions like psoriasis. This can be overcome by application of low concentration capsaicin multiple times or high concentration once to desensitize the area of application. Previously only two strengths were available, $0.075 \%$ for neuropathic pain and $0.025 \%$ for arthritic pain, which are given up to four times daily. The Food and Drug Administration (FDA) approved high-concentration capsaicin 8\% patch (Qutenza) for neuropathic pain, which can provide pain relief for up to 12 weeks after a single 60-minute application [25].

Topical lidocaine: Lidocaine is available in various cream formulations and as patches. The only FDA-approved use of 5\% lidocaine is for postherpetic neuralgia for which it is applied 12 hours daily. With only mild skin irritation as a side effect, it can be used for various other neuropathic pain conditions.

Opioid analgesics: Opioid analgesics are considered for managing severe pain or failed response to other treatments in the elderly. Although the short-term efficacy of opioid analgesics in chronic pain in the elderly is established, [26] limited evidence is available regarding the longterm efficacy because of the discontinuation of opioid therapy due to various poorly tolerable side effects [27]. Opioid analgesics are classified into strong opioids like morphine, hydromorphone, oxycodone, fentanyl, and buprenorphine and weak opioids such as tapentadol and tramadol. Weaker opioids, tapentadol, and tramadol have a dual mechanism of action, as both mu-opioid agonists and norepinephrine reuptake inhibitors. Tramadol was approved by the FDA but caution is warranted in its use due to adverse effects like sedation, cognitive 
impairment, and interaction with other medications. Due to its strong serotonin reuptake inhibitory action, caution must also be taken particularly when prescribing tramadol to an elderly patient on selective serotonin reuptake inhibitors to avoid serotonin syndrome.

Tapentadol was also approved for this use by the FDA in 2008. Unlike tramadol, tapentadol has only weak effects on serotonin reuptake and has no active metabolites, but tapentadol must be used with caution in the elderly because of the risk of causing severe hypotension, respiratory depression, and sedation. Randomized placebo and active-controlled studies done on elderly patients older than 75 years with chronic pain showed the similar efficacy of tapentadol ER and oxycodone controlled-release groups; also, more GI adverse effects were reported in oxycodone groups than tapentadol [28]. The use of strong opioids for managing pain in the elderly warrants caution because of the increased half-life of the active drug metabolites. Both short and long-acting agents have similar efficacy but a randomized crossover study showed that transdermal fentanyl was preferred by patients to sustained release morphine not only because it provided better pain relief but also because of the high incidence of constipation in the morphine group [29].

A prerequisite before initiation of opioid therapy is the evaluation of risk versus benefit in view of its adverse effects, and efforts to reduce risks are mandatory. Administration of opioids in the elderly is done on a trial basis to titrate the effective dose reaching the therapeutic goal with minimal adverse effects, starting with the lowest possible dose and gradual titration.

Respiratory depression is one of the major concerns of opioid therapy, though tolerance to this effect develops rapidly. Respiratory depression is particularly common in patients who increase their doses rapidly, patients using drugs like methadone with variable pharmacokinetics, patients with concomitant use of drugs like benzodiazepines or barbiturates. Elderly patients in particular with hepatic and renal dysfunction are at increased risk due to resultant drug accumulation, which warrants regular monitoring of parameters like glomerular filtration and dose adjustments [30, 31]. With due course of time, tolerance develops to the majority of the side effects of opioids (except constipation due to gastric hypomotility) like respiratory depression, sedation, nausea, and vomiting. Until the development of tolerance, patients are managed with combined administration of antiemetics and usage of assistive devices. Complications of long-term usage of opioids include suppressed production of pituitary, gonadal, hypothalamic, and adrenal hormones manifesting as depression, fatigue, and decreased libido [1]. With prolonged therapy, opioid abuse is another major concern. Every patient should be assessed for risk factors related to the potential abuse with available tools like opioid Risk Tool (ORT) and the SOAPP-R (revised Screener and Opioid Assessment for Patients with Pain) [32]. Although clinicians must remain vigilant about the possibility of misuse or abuse of opioid agents in all patients, older age is generally associated with a relatively lower risk for opioid misuse and abuse [33].

Anticonvulsants: Antiepileptic drugs such as carbamazepine, gabapentin, and pregabalin are mainly used for neuropathic pain. In elderly patients with renal impairment, dose adjustment of gabapentin and pregabalin is required. Carbamazepine currently is the first line therapy for neuralgia. Gabapentin and pregabalin are recommended to be taken in short courses (two to four months) for certain types of neuropathic pain including diabetic neuropathy, central neuropathic pain after spinal cord injury, postherpetic neuralgia, and fibromyalgia. A metaanalysis study assessing $300 \mathrm{mg}$ of pregabalin daily for neuropathic pain showed $50 \%$ reduction in pain [34]. Gabapentin dosage must be carefully titrated starting with 100-300 mg daily, up to a maximum dose of $3600 \mathrm{mg}$ [35].

Serotonin-norepinephrine reuptake inhibitors (SNRIs): SNRIs such as venlafaxine and duloxetine can be used for neuropathic pain in the elderly. SNRIs are generally well tolerated but side effects include hyponatremia, giddiness, nausea, and abdominal pain. Venlafaxine is given at a daily dose of $37.5 \mathrm{mg}$, which can be increased up to $300 \mathrm{mg}$ particularly in elderly patients with associated depression. But its use in the elderly is still under controversy, and 
results of a few studies actually limit its use due to hypertensive episodes. On the contrary, duloxetine has no cardiovascular effects and it showed a $50 \%$ reduction in diabetic neuropathic pain compared to placebos [36]. Dose titration must be done carefully as most of the side effects are dose-dependent.

Tricyclic antidepressants (TCAs): Tricyclic antidepressants such as amitriptyline, desipramine, and nortriptyline are effective in the treatment of postherpetic neuralgia and painful peripheral diabetic neuropathy, but tertiary tricyclics like amitriptyline is usually avoided in elderly patients in view of a higher incidence of side effects. Nortriptyline is usually given at a starting daily dose of $25 \mathrm{mg}$ up to $200 \mathrm{mg}$ if an associated depression is present. Adverse effects include both due to anticholinergic and noradrenergic effects. QTc prolongation is a major complication, so an electrocardiogram screening is a must before starting tricyclic therapy [37].

Benzodiazepines: Use of benzodiazepines for chronic pain in the elderly is limited by its adverse effects like tendency to falls and cognitive decline. It was found that $10 \%-20 \%$ of the falls in the elderly are due to the use of benzodiazepines [38]. Both long and short-acting benzodiazepines are included on the Beers list [39] despite the evidence of both increasing the risk of falls in the elderly. But considering the above facts, the use of benzodiazepines is usually limited in the elderly to conditions where spasm muscle, anxiety, and pain coexist.

Muscle relaxants: Baclofen, a GABA-B agonist is recommended as a second-line agent for neuropathic pain, usually used in patients with spasticity due to central nervous system injury, demyelinating conditions, and other neuromuscular disorders. The initial dose has to be low, gradually increased to minimize the side effects like dizziness, somnolence, and gastrointestinal symptoms and slowly tapered before discontinuation to avoid delirium and seizures [40].

\section{Other Agents}

Calcitonin acting by an unknown mechanism is effective as a second-line agent for neuropathic pain and also shown to be effective in treating pain due to osteoporotic vertebral compression fractures and in patients with bone metastases [41, 42]. Usual side effects include hypersensitivity reactions and nausea. In addition, patients require regular monitoring of calcium and phosphate levels. Another group of agents also useful in pain due to cancer metastases are bisphosphonates particularly pamidronate and clodronate. These agents must be used with caution in elderly patients with renal failure, and also there is an occasional risk of esophagitis and hypocalcemia [43].

\section{Newer Therapies}

Cannabinoids: Medicinal use of cannabinoids is legal and are now available by prescription. Cannabinoids act on two classic receptors CB1r and CB2r. CB1 receptors are found in the central and peripheral nervous systems, bone, heart, liver, lung, vascular endothelium, and reproductive system while CB2 receptors are primarily found in the immune system, also at lower levels in the nervous system. CB1 receptors are the ones responsible for psychotomimetic adverse effects and addiction, because of which selective CB2r agonists are being preferred [44]. A buccal spray preparation Sativex is available from GW Pharmaceuticals Ltd, UK, which contains both psychoactive (tetrahydrocannabinol) and non-psychoactive (cannabidiol) extract from the cannabis plant. It is used for the treatment of neuropathic pain in multiple sclerosis patients. Other preparations available are synthetically derived nabilone and dronabinol, which are taken orally. Both these preparations are primarily used for chemotherapy-induced nausea and vomiting with only minimal analgesic action. A recent study on elderly patients greater than 65 years of age using cannabinoids for various conditions showed a marked reduction in 
pain and also better safety profile compared to opioids, but the safety of long-term therapy is not established. Common side effects include euphoria, anxiety, psychosis, sedation, dizziness, cognitive effects, tachycardia, palpitations, and postural hypotension. Due to narrow therapeutic index, elderly in particular are at higher risk of dysphoric effects of cannabinoids. In addition, the elderly must be warned against potential dangers during driving because of the effects of cannabinoids on reaction time and motor control $[45,46]$.

Micronized NSAIDs: Micronized NSAIDs are developed using nanotechnology to increase the surface area of drug and thus their absorption, providing comparable efficacy to conventional formulations at lower doses with minimized adverse effects. Previously FDA-approved two micronized NSAIDs, diclofenac (Zorvolex) and indomethacin (Tivorbex) for the treatment of mild-moderate pain in adults. FDA approved another drug meloxicam (Vivlodex ${ }^{\mathrm{TM}}$ ) for osteoarthritis following data from a phase 3 study of 402 patients with osteoarthritis pain in the hip or knee, the results of which showed that micronized meloxicam achieved efficacy at $33 \%$ lower doses than currently available meloxicam products [47].

\section{Upcoming Novel Therapies}

Target opioids: Several newer opioid agents with better safety profiles such as target opioid and mixed opioid agonists are currently under development. Activation of the mu-opioid receptor (MOR receptor) results in G-protein mediated analgesic effect of opioids; the receptor activation also results in recruitment of B-arrestin which is responsible for respiratory depression and various other side effects of opioids. TRV130 (Oliceridine) is a new $\mu$-receptor G-protein pathway selective ( $\mu$-GPS) modulator with low $\beta$-arrestin recruitment and thus acceptable safety profile. Studies showed that TRV130 showed similar efficacy to that of morphine but with a wider therapeutic window and better safety profile [48]. Efficacy of TRV130 for chronic pain has not been studied until now and is also not yet approved by FDA even for acute pain. Most of the traditionally available opioids are MOP agonists; a mixed opioid agonist with a target combination of $\mathrm{mu}$ (MOP) and nociceptin/orphanin FQ peptide (NOP) receptors is currently being studied [49]. Two new MOP/NOP ligands SR16435 and BU08028 compared with morphine in mouse models demonstrated higher nociceptive potential with reduced development of tolerance to analgesia [50]. A preclinical study comparing BU08028 with MOP receptor agonists in nonhuman primates showed that BU08028 did not cause any cardiovascular side effects or respiratory depression even at 10- to 30-fold higher doses. Unlike with morphine, there is no development of physical dependence with BU08028. The mixed MOP/NOP agonist still needs further studies in humans, but data currently available is showing better efficacy and abuse free potential.

\section{Conclusions}

Treating chronic pain in the elderly population still poses many challenges. A multidisciplinary approach using multicomponent strategies is needed as the most efficacious and safest therapeutic option for this population. With the introduction of targeted therapy and newer emerging modalities of pain control in the clinical practice, we hope managing chronic pain becomes easy in the near future. Prescribing physicians should start treating the geriatric population with the lowest possible, tolerable doses aimed at causing less adverse effects and improving the quality of life.

\section{Additional Information \\ Disclosures}

Conflicts of interest: In compliance with the ICMJE uniform disclosure form, all authors declare the following: Payment/services info: All authors have declared that no financial 
support was received from any organization for the submitted work. Financial relationships: All authors have declared that they have no financial relationships at present or within the previous three years with any organizations that might have an interest in the submitted work. Other relationships: All authors have declared that there are no other relationships or activities that could appear to have influenced the submitted work.

\section{References}

1. American Geriatrics Society Panel on Pharmacological Management of Persistent Pain in Older Persons: Pharmacological management of persistent pain in older persons . J Am Geriatr Soc. 2009, 57:1331-1346. 10.1111/j.1532-5415.2009.02376.x

2. Mohamed Zaki LR, Hairi NN: Chronic pain and pattern of health care uti-lization among Malaysian elderly population: National Health and Mor־bidity Survey III (NHMS III, 2006). Maturitas. 2014, 79:435-441. 10.1016/j.maturitas.2014.08.014

3. Bauer H, Emeny RT, Baumert J, Ladwig KH: Resilience moderates the association between chronic pain and depressive symptoms in the elderly. Eur J Pain. 2016, 20:1253-1265. 10.1002/ejp.850

4. Zanocchi M, Maero B, Nicola E, et al.: Chronic pain in a sample of nursing home residents: prevalence, characteristics, influence on quality of life (QoL). Arch Gerontol Geriatr. 2008, 47:121-128. 10.1016/j.archger.2007.07.003

5. Shega JW, Dale W, Andrew M, Paice J, Rockwood K, Weiner DK: Persistent pain and frailty: a case for homeostenosis. J Am Geriatr Soc. 2012, 60:113-117. 10.1111/j.15325415.2011.03769.x

6. Dziechciaż M, Balicka-Adamik L, Filip R: The problem of pain in old age . Ann Agric Environ Med. 2013, 20:35-8.

7. Ruoff GE: Challenges of managing chronic pain in the elderly . Semin Arthritis Rheum. 2002, 32:43-50.10.1053/sarh.2002.37214

8. Dubois MY: Practical management of pain, third edition . J Pain Symptom Manage. 2001, 21:529-530. 10.1016/S0885-3924(01)00293-7

9. Broe GA: The neuroepidemiology of old age. The Clinical Neurology of Old Age. Tallis R (ed): Wiley, Chichester, UK; 1989. 50-80.

10. Herr KA, Garand L: Assessment and measurement of pain in older adults . Clin Geriatr Med. 2001, 17:457-vi.

11. Ngamkham S, Vincent C, Finnegan L, Holden JE, Wang ZJ, Wilkie DJ: The McGill Pain Questionnaire as a multidimensional measure in people with cancer: an integrative review. Pain Manag Nurs. 2012, 13:27-51. 10.1016/j.pmn.2010.12.003

12. Lints-Martindale AC, Hadjistavropoulos T, Lix LM, Thorpe L: A comparative investigation of observational pain assessment tools for older adults with dementia. Clin J Pain. 2012, 28:226237. 10.1097/AJP.0b013e3182290d90

13. Morone NE, Greco CM: Mind-body interventions for chronic pain in older adults: a structured review. Pain Med. 2007, 8:359-375. 10.1111/j.1526-4637.2007.00312.x

14. Weiner DK, Ernst E: Complementary and alternative approaches to the treatment of persistent musculoskeletal pain. Clin J Pain. 2004, 20:244-255.

15. Davies E, Higginson IJ: Better Palliative Care for Older People. Davies E, Higginson IJ (ed): WHO Regional Office for Europe, Copenhagen; 2004.

16. Towheed TE, Maxwell L, Judd MG, Catton M, Hochberg MC, Wells G: Acetaminophen for osteoarthritis. Cochrane Database Syst Rev. 2006, 25:004257.

10.1002/14651858.CD004257.pub2

17. Chou R, Huffman LH: Medications for acute and chronic low back pain: a review of the evidence for an American Pain Society/American College of Physicians clinical practice guideline. Ann Intern Med. 2007, 147:505-514. 10.7326/0003-4819-147-7-200710020-00008

18. Wegman A, van der Windt D, van Tulder M, Stalman W, de Vries T: Nonsteroidal antiinflammatory drugs or acetaminophen for osteoarthritis of the hip or knee? A systematic review of evidence and guidelines. J Rheumatol. 2004, 31:344-354.

19. Franceschi M, Scarcelli C, Niro V, et al.: Prevalence, clinical features and avoidability of adverse drug reactions as cause of admission to a geriatric unit: a prospective study of 1756 patients. Drug Saf. 2008, 31:545-556. 
20. Lanza F, Rack MF, Doucette M, Ekholm B, Goldlust B, Wilson R: An endoscopic comparison of the gastroduodenal injury seen with salsalate and naproxen. J Rheumatol. 1989, 16:15701574.

21. Setakis E, Leufkens HG, van Staa TP: Changes in the characteristics of patients prescribed selective cyclooxygenase 2 inhibitors after the 2004 withdrawal of rofecoxib. Arthritis Rheum. 2008, 59:1105-1111. 10.1002/art.23925

22. Trelle S, Reichenbach S, Wandel S, et al.: Cardiovascular safety of non-steroidal antiinflammatory drugs: network meta-analysis. BMJ. 2011, 342:c7086. 10.1136/bmj.c7086

23. Tugwell PS, Wells GA, Shainhouse JZ: Equivalence study of a topical diclofenac solution (pennsaid) compared with oral diclofenac in symptomatic treatment of osteoarthritis of the knee: a randomized controlled trial. J Rheumatol. 2004, 31:2002-12.

24. Arnstein PM: Evolution of topical NSAIDs in the guidelines for treatment of osteoarthritis in elderly patients. Drugs Aging. 2012, 29:523-531. 10.2165/11631550-000000000-00000

25. Anand P, Bley K: Topical capsaicin for pain management: therapeutic potential and mechanisms of action of the new high-concentration capsaicin 8\% patch. Br J Anaesth. 2011, 107:490-502. 10.1093/bja/aer260

26. Papaleontiou M, Henderson CR Jr, Turner BJ, Moore AA, Olkhovskaya Y, Amanfo L, Reid MC: Outcomes associated with opioid use in the treatment of chronic noncancer pain in older adults: a systematic review and meta-analysis. J Am Geriatr Soc. 2010, 58:1353-69. 10.1111/j.1532-5415.2010.02920.x

27. Reid MC, Henderson CR Jr, Papaleontiou M, et al.: Characteristics of older adults receiving opioids in primary care: treatment duration and outcomes. Pain Med. 2010, 11:1063-71. 10.1111/j.1526-4637.2010.00883.x

28. Biondi DM, Xiang J, Etropolski M, Moskovitz B: Tolerability and efficacy of tapentadol extended release in elderly patients $\$ 75$ years of age with chronic osteoarthritis knee or low back pain. J Opioid Manag. 2015, 11:393-403.

29. Allan L, Hays H, Jensen NH, de Waroux BL, Bolt M, Donald R, Kalso E: Randomised crossover trial of transdermal fentanyl and sustained release oral morphine for treating chronic noncancer pain. BMJ. 2001, 322:1154.

30. Dowell D, Haegerich TM, Chou R: CDC guideline for prescribing opioids for chronic pain - the United States, 2016. MMWR Recomm Rep. 2016, 65:1-49. 10.15585/mmwr.rr6501e1

31. Carbonara GM: Opioids in patients with renal or hepatic dysfunction . Pract Pain Manag. 2011, 8:Accessed: September 13, 2018:

https://www.practicalpainmanagement.com/treatments/pharmacological/opioids/opioidspatients-renal-hepatic-dysfunction.

32. Henrie-Barrus P, Averill LA, Sudweeks RR, Averill CL, Mota N: Development and preliminary validation of the opioid abuse risk screener. Health Psychol Open. 2016, 3: 2055102916648995. 10.1177/2055102916648995

33. Edlund MJ, Steffick D, Hudson T, Harris KM, Sullivan M: Risk factors for clinically recognized opioid abuse and dependence among veterans using opioids for chronic noncancer pain. Pain. 2007, 129:355-362. 10.1016/j.pain.2007.02.014

34. Snedecor SJ, Sudharshan L, Cappelleri JC, Sadosky A, Desai P, Jalundhwala Y, Botteman M: Systematic review and meta-analysis of pharmacological therapies for pain associated with postherpetic neuralgia and less common neuropathic conditions. Int J Clin Pract. 2014, 68:900-918. 10.1111/ijcp.12411

35. Oneschuk D, al-Shahri MZ: The pattern of gabapentin use in a tertiary palliative care unit . J Palliat Care. 2003, 19:185-187.

36. Goldstein DJ, Lu Y, Detke MJ, Lee TC, Iyengar S: Duloxetine vs. placebo in patients with painful diabetic neuropathy. Pain. 2005, 116:109-118. 10.1016/j.pain.2005.03.029

37. Sindrup SH, Otto M, Finnerup NB, Jensen TS: Antidepressants in the treatment of neuropathic pain. Basic Clin Pharmacol Toxicol. 2005, 96:399-409. 10.1111/j.17427843.2005.pto_96696601.x

38. Blazer D, Hybels C, Simonsick E, Hanlon JT: Sedative, hypnotic, and antianxiety medication use in an aging cohort over ten years: a racial comparison. J Am Geriatr Soc. 2000, 48:10731079. 10.1111/j.1532-5415.2000.tb04782.x

39. American Geriatrics Society 2015 Beers Criteria Update Expert Panel: American geriatrics society 2015 updated beers criteria for inappropriate medication use in older adults. J Am Geriatr Soc. 2015, 63:2227-2246. 10.1111/jgs.13702 
40. Fromm GH: Baclofen as an adjuvant analgesic. J Pain Symptom Manage. 1994, 9:500-509. 10.1016/0885-3924(94)90111-2

41. Lyritis GP, Paspati I, Karachalios T, Ioakimidis D, Skarantavos G, Lyritis PG: Pain relief from nasal salmon calcitonin in osteoporotic vertebral crush fractures. A double-blind, placebocontrolled clinical study. Acta Orthop Scand Suppl. 1997, 275:112-114.

42. Roth A, Kolaric K: Analgetic activity of calcitonin in patients with painful osteolytic metastases of breast cancer. Results of a controlled randomized study. Oncology. 1986, 43:283-287. 10.1159/000226383

43. Gralow J, Tripathy D: Managing metastatic bone pain: the role of bisphosphonates . J Pain Symptom Manage. 2007, 33:462-472. 10.1016/j.jpainsymman.2007.01.001

44. Howlett AC, Barth F, Bonner TI, et al.: International Union of Pharmacology. XXVII. Classification of cannabinoid receptors. Pharmacol Rev. 2002, 54:161-202.

45. Onaivi ES, Ishiguro H, Gong JP, et al.: Brain neuronal CB2 cannabinoid receptors in drug abuse and depression: from mice to human subjects. PLoS One. 2008, 3:1640-1610.

10.1371/journal.pone.0001640

46. Nano-formulated NSAIDs: a new dawn for safe use . (2015). Accessed: September 13, 2018: https://www.pharmacytimes.com/contributor/jeffrey-fudin/2015/09/nano-formulated-nsaidsa-new-dawn-for-safe-use.

47. Van Sickle MD, Duncan M, Kingsley PJ, et al.: Identification and functional characterization of brainstem cannabinoid CB2 receptors. Science. 2005, 310:329-332. 10.1126/science.1115740

48. Viscusi ER, Webster L, Kuss M, et al.: A randomized, phase 2 study investigating TRV130, a biased ligand of the $\mu$-opioid receptor, for the intravenous treatment of acute pain. Pain. 2016, 157:264-272. 10.1097/j.pain.0000000000000363

49. Sukhtankar DD, Zaveri NT, Husbands SM, Ko M-C: Administered bifunctional nociceptin/orphanin FQ peptide receptor/ $\mu$-opioid receptor ligands in mouse models of neuropathic and inflammatory pain. J Pharmacol Exp Ther. 2013, 346:11-22. 10.1124/jpet.113.203984

50. Ding H, Czoty PW, Kiguchi N, et al.: A novel orvinol analog, BU08028, as a safe opioid analgesic without abuse liability in primates. Proc Natl Acad Sci U S A. 2016, 113:5511-5518. 10.1073/pnas.1605295113 